\title{
Design and implementation of an improved chilled water glycol system for GeMS: CANOPUS thermal enclosures
}

Gaston Gausachs, Matthieu Bec, Ramon Galvez, Chas Cavedoni, Vicente Vergara, et al.

Gaston Gausachs, Matthieu Bec, Ramon Galvez, Chas Cavedoni, Vicente Vergara, Herman Diaz, German Fernandez, "Design and implementation of an improved chilled water glycol system for GeMS: CANOPUS thermal enclosures," Proc. SPIE 7735, Ground-based and Airborne Instrumentation for Astronomy III, 77356D (20 July 2010); doi: 10.1117/12.857338

SPIE Event: SPIE Astronomical Telescopes + Instrumentation, 2010, San Diego, California, United States 


\title{
Design and Implementation of an Improved Chilled Water Glycol System for GeMS - CANOPUS Thermal Enclosures
}

\author{
Gaston Gausachs ${ }^{\mathrm{a}}$, Matthieu Bec ${ }^{\mathrm{a}}$, Ramon Galvez ${ }^{\mathrm{a}}$, Chas Cavedoni ${ }^{\mathrm{b}}$, Vicente Vergara ${ }^{\mathrm{a}}$, \\ Herman Diaz ${ }^{\mathrm{a}}$, German Fernandez ${ }^{\mathrm{c}}$ \\ ${ }^{a}$ Gemini Observatory, Colina El Pino s/n, La Serena, Chile \\ ${ }^{\mathrm{b}}$ Gemini Observatory, 670 North A'Ohoku Place, Hilo, Hawaii, U.S.A. \\ ${ }^{\mathrm{c} C}$ Complejo Astronómico El Leoncito, Av. España Sur 1512, San Juan, Argentina
}

\begin{abstract}
CANOPUS is the facility instrument for the Gemini Multi Conjugate Adaptive Optics System (GeMS) wherein all the adaptive optics mechanisms and associated electronic are tightly packed. At an early stage in the pre-commissioning phase Gemini undertook the redesign and implementation of its chilled Ethylene Glycol Water (EGW) cooling system to remove the heat generated by the electronic hardware. The electronic boards associated with the Deformable Mirrors (DM) represent the highest density heat yielding components in CANOPUS and they are also quite sensitive to overheating. The limited size of the two electronic thermal enclosures (TE) requires the use of highly efficient heat exchangers (HX) coupled with powerful yet compact DC fans.

A systematic approach to comply with all the various design requirements brought about a thorough and robust solution that, in addition to the core elements (HXs and fan), makes use of features such as high performance vacuum insulated panels, vibration mitigation elements and several environment sensors. This paper describes the design and implementation of the solution in the lab prior to delivering CANOPUS for commissioning.
\end{abstract}

Keywords: Chilled water glycol system, electronic cooling, AO.

\section{INTRODUCTION}

CANOPUS is part of the larger Multi Conjugate Adaptive Optics System (GeMS) due for commissioning in the end of 2010 at Gemini Observatory, Southern Operations Center at Cerro Pachon, Chile. CANOPUS, originally called Adaptive Optics Module (AOM) was built at EOSt facilities in Tucson, AZ, and later shipped to Gemini in 2006 for final integration. CANOPUS objective is to use three Deformable Mirrors (DM) conjugated at different altitudes to accurately cancel the atmospheric turbulence and deliver science quality images to the Gemini South Adaptive Optics Imager (GSAOI) and FLAMINGOS 2.

The Deformable Mirror Electronics (DME) account for a large fraction of the waste heat produced inside CANOPUS TE. Despite an original design requirement calling for a chilled water glycol system, the delivered solution was deemed insufficient to cope with the existing operating conditions. As a result, Gemini decided to tackle this issue by completely redesigning the electronic cooling system. The design effort culminated in a complete solution for cooling not only the DME but also the Laser Guide Star Wave Front Sensors (LGSWFS) CCD housing and the camera for slow focus sensor running on a separate lower pressure-lower risk circuit.

In addition to the baseline cooling provided by the air-to-liquid Heat Exchangers the solution includes special control features to prevent condensation and overheating under various possible scenarios. New electronics and software capabilities were built into the cooling system.

Ground-based and Airborne Instrumentation for Astronomy III, edited by lan S. McLean,

Suzanne K. Ramsay, Hideki Takami, Proc. of SPIE Vol. 7735, 77356D - @ 2010

SPIE · CCC code: 0277-786X/10/\$18 - doi: 10.1117/12.857338 


\section{INITIAL CONDITIONS}

CANOPUS consists of three large enclosures: the central AOB enclosure containing all the opto-mechanical elements and one Thermal Enclosure (TE) on each side where the electronics, power, computing and network equipment are cooled. As in all the instruments TE in use on the telescope, the cooling relies on chilled 40/60 Ethylene Glycol Water (EGW) circulating through air-to-liquid heat exchangers or, in some cases, cold plates.

Under maximum power conditions CANOPUS outputs almost $4.1 \mathrm{~kW}$ of waste heat. The facility chilled EGW constrains the coolant flow rate to a maximum of $12 \mathrm{lpm}$ at 0 to $10 \mathrm{C}$. Further requirements listed in Gemini Internal Control Documents (ICD), describe design guidelines such as a maximum internal TE temperature of $20 \mathrm{C}$.

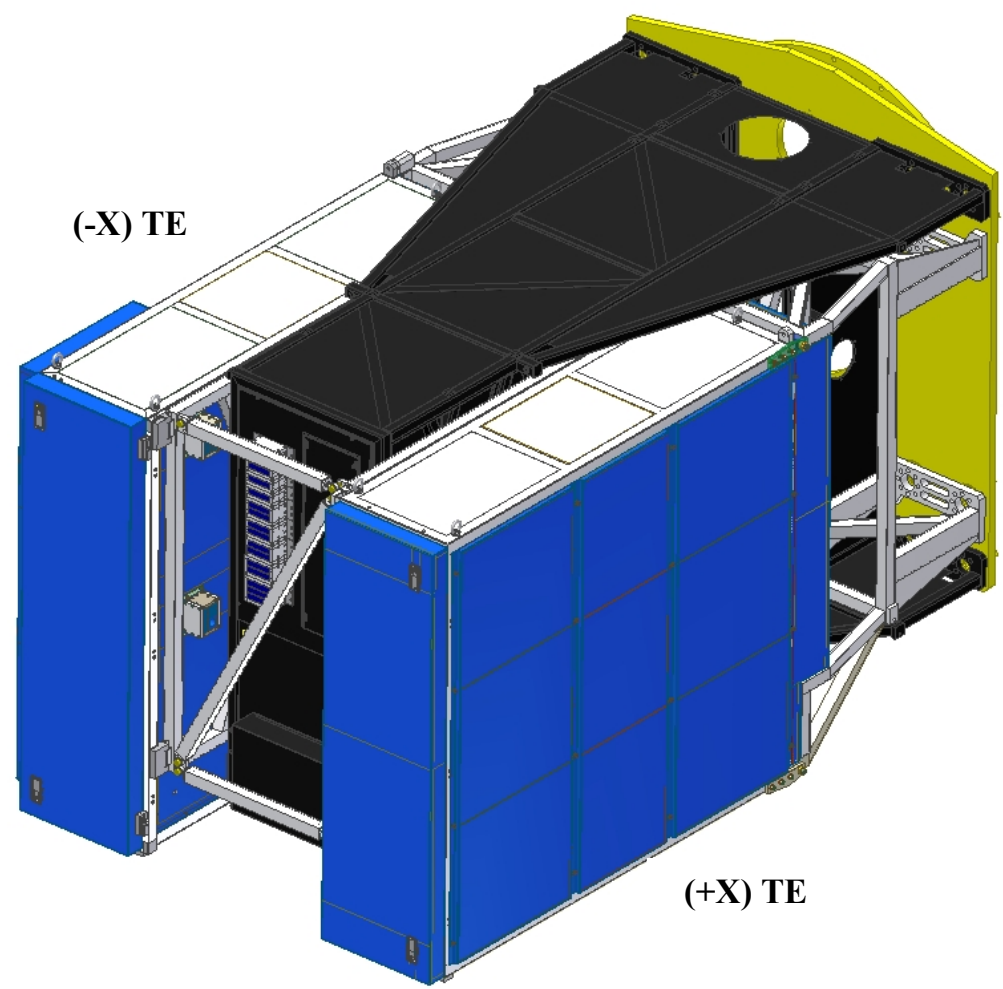

Figure 1. CANOPUS AOB in black and both TE and support frame in white and blue.

The space envelope for the instrument is critical since CANOPUS must coexist on the telescope instrument cluster without interfering with neighboring systems during operation. This tight envelope restricts the available internal volume and makes for closely packed electronics inside the TE. The insulating material originally selected by the vendor are vacuum insulated panels (VIP) made by Nanopore because they offer the best performance for a 12 and $25 \mathrm{~mm}$ thickness.

\section{SYSTEM OVERVIEW}

\subsection{HEAT EXCHANGERS \& FANS}

\subsubsection{Selection}

The cooling loops in each CANOPUS TE are arranged in accordance with the grouping of electronic components. Three Lytron ES0714 heat exchangers were selected for the (-X) TE and two smaller Lytron ES0710 for the (+X) TE. Lytron heat exchangers offer an improved cooling capacity over the previous heat exchangers due to their flat tubing with internal fins. 


\begin{tabular}{|c|c|c|c|c|c|c|c|c|}
\hline \multicolumn{2}{|c|}{ Location within CANOPUS } & $\begin{array}{l}\text { Load } \\
\text { [W] }\end{array}$ & $\begin{array}{l}\text { Fan } \\
\text { [W] }\end{array}$ & $\begin{array}{l}\text { Total } \\
\text { [W] }\end{array}$ & $\begin{array}{l}\text { Coolant } \\
\text { [GPM] }\end{array}$ & $\begin{array}{c}\text { Fan } \\
\text { [CFM] }\end{array}$ & $\begin{array}{c}\text { Cooling } \\
\%\end{array}$ & $\begin{array}{c}\text { Fan } \\
\%\end{array}$ \\
\hline \multirow{3}{*}{-X Enclosure } & -Y, DME 0/9 & 1058 & 82 & 1140 & 0.5 & 300 & 102.5 & 278 \\
\hline & $-Y, D M E 4.5$ & 815 & 82 & 897 & 0.5 & 300 & 130 & 278 \\
\hline & $+\mathrm{Y}$ Bay $(\mathrm{HV})$ & 721 & 82 & 803 & 0.5 & 300 & 146 & 278 \\
\hline \multirow{2}{*}{ +X Enclosure } & -Y Bay (RTC) & 309 & 27 & 336 & 0.5 & 112 & 171 & 131 \\
\hline & +Y Bay (VME) & 491 & 27 & 518 & 0.5 & 112 & 111 & 131 \\
\hline \multicolumn{4}{|c|}{ TOTAL Heat Exchangers } & 3694 & 2.5 & & & \\
\hline
\end{tabular}

\begin{tabular}{|c|c|c|c|c|c|}
\hline \multicolumn{2}{|c|}{ Location within CANOPUS } & $\begin{array}{l}\text { Load } \\
\text { [W] }\end{array}$ & $\begin{array}{l}\text { Total } \\
\text { [W] }\end{array}$ & $\begin{array}{l}\text { Coolant } \\
\text { [GPM] }\end{array}$ & $\begin{array}{c}\text { Cooling } \\
\%\end{array}$ \\
\hline \multirow{2}{*}{$\mathrm{AOB}$} & CCD $\times 5$ & 75 & 75 & 0.1 & 180 \\
\hline & APOGEE/SFS & 15 & 15 & 0.02 & \\
\hline \multicolumn{2}{|c|}{ SDSU Controller Enclosure } & 300 & 300 & 0.25 & \\
\hline \multicolumn{3}{|c|}{ TOTAL Cold Plates } & 390 & 0.37 & \\
\hline
\end{tabular}

\begin{tabular}{|l|r|c|}
\hline \multicolumn{1}{|c|}{ Cooling System } & $\begin{array}{c}\text { Load } \\
\text { [W] }\end{array}$ & $\begin{array}{c}\text { Coolant } \\
\text { [GPM] }\end{array}$ \\
\hline Heat Exchangers & 3694 & 2.50 \\
\hline Cold Plates & 390 & 0.37 \\
\hline TOTAL CANOPUS & $\mathbf{4 0 8 4}$ & $\mathbf{2 . 8 7}$ \\
\hline
\end{tabular}

Figure 2. Summary shows the different cooling circuits in CANOPUS with associated heat load and margin of safety.

While the original design worked with AC fans, it was decided to use DC brushless fans as they provide higher static pressure and airflow in the same form factor. The fans on the Lytron ES0714 are ebmpapst model DV6224/2 TDA-824 24 DCV while the Lytron ES0710 are fitted with ebmpapst fan model 4184NXH 24 DCV. The former have adjustable speed through the software while the other fans are adjusted once, in the field, and set at a constant speed.

Selection of the heat exchangers and fans pairs was made to match the initial conditions listed before and the operating conditions at the Cerro Pachon summit $(2750 \mathrm{~m})$. Altitude makes the air thinner and the lower mass flow decreases the fans cooling performance. The 0/9 DME generates most of the heat inside the (-X) TE components and its estimated cooling margin is the lowest at roughly $15 \%$; all the other components having higher margin.

\subsubsection{Ducted design}

To improve airflow on the DME racks the cooling solution was built to provide both of them with dedicated heat exchangers and fans placed inside a ducted air path. The flow is directed across each board and avoids wasting cool air to non heated components. A partial bulkhead was created between the (-X) TE Cable bay and High Voltage bay to avoid short circuiting the airflow.

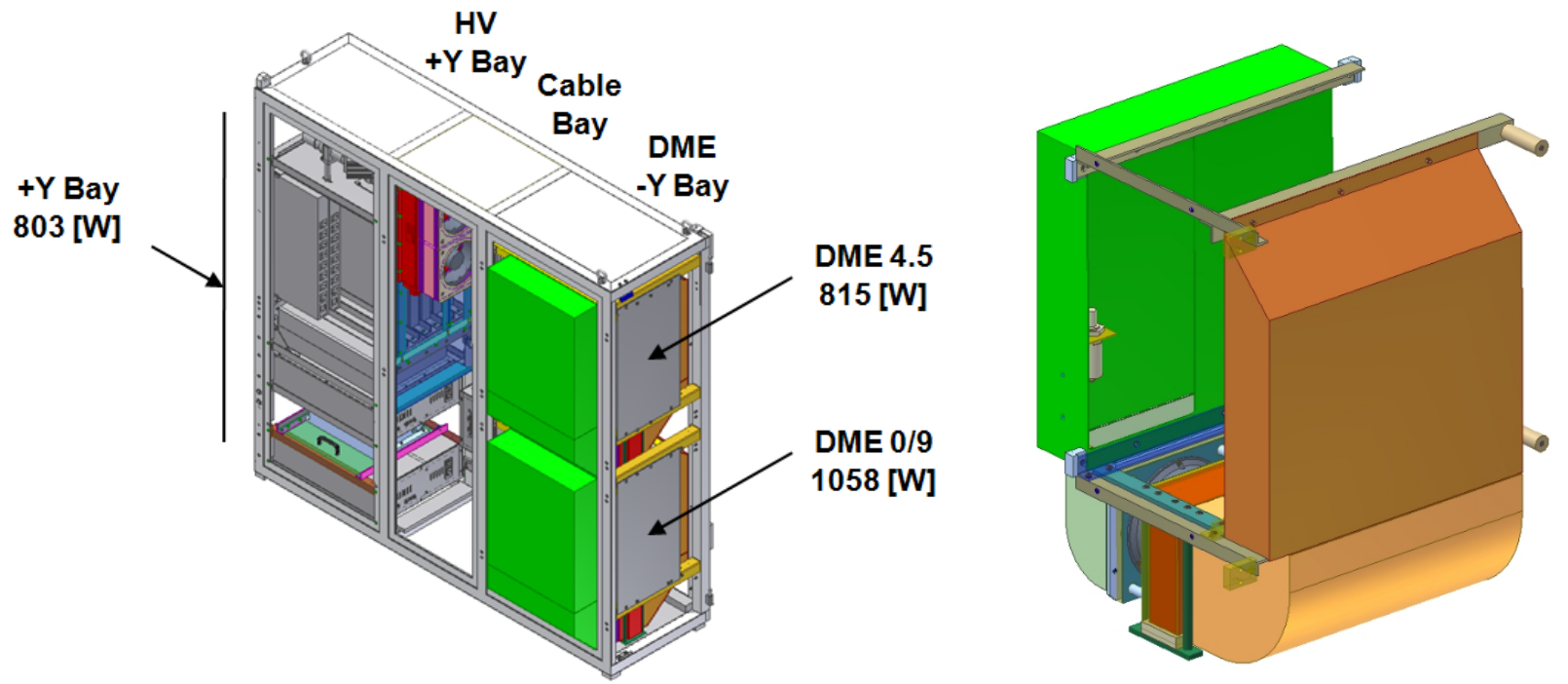

Figure 3. (-X) TE with covers removed showing the distinct cooling circuits (DME4.5, DME 0/9 and HV) (left). Detail of the DME cooling ducted design (right) 


\subsubsection{Vibration Mitigation}

As an AO system, CANOPUS optics is sensitive to vibration that fall within its operating bandwidth. From the onset of the design it was clear that any change of the hardware needed to add the least amount of vibrating energy. Early tests showed it was possible to operate more powerful DC fans attached on a test rig to the TE frame without a noticeable effect to the AO performance. The final design included with the ability to change the mounting and, at the same time, dampening material of the larger fans inside the $(-X) \mathrm{TE}$; the smaller $(+\mathrm{X}) \mathrm{TE}$ fans are hard mounted instead.

\subsection{COOLANT MANIFOLDS}

\subsubsection{Primary Cooling}

In order to meet the maximum allowable pressure drop across the coolant circuit (10 psi) each individual heat exchanger was connected to a main supply and return coolant manifold in parallel. In total five heat exchanger circuits plus one circuit for the SDSU Controller coldplates constitute the primary cooling circuit. The tests currently underway have demonstrated that the pressure drop is no higher than 8 psi. Each sub-circuit can be individually purged of air through the quick disconnects at each hose.

Due to its size, the manifold is installed directly under the (-X) TE. Two Proteus 4012 temperature, flow and pressure sensors provide local display and remote status of these parameters on the main supply and return circuits. Each of the six branches has a Proteus 804 flow sensor with remote readout, in line with a needle valve for flow adjustments. The ($\mathrm{X}$ ) TE sub circuits may be shut off remotely via a solenoid valve whenever the risk of condensation arises inside that particular TE. A dew point sensor measuring ambient air complements the sensing suite of the manifold.

\subsubsection{Secondary Cooling}

The Laser Guide Star Wave Front Sensor has five CCD, each with independent cooling heads. The sensitive nature of the CCD and the lower heat output calls for a smaller cooling circuit that runs at a lower flow and pressure. The secondary cooling enclosure is essentially a manifold with a single flow meter distributing the flow to the five CCD plus the Apogee camera. The secondary cooling loop will connect to a dedicated coolant line once CANOPUS is installed on the telescope.

\subsection{DRY AIR MANIFOLD}

A requirement to keep a low moisture environment and avoid condensation on critical high voltage (+/- 370V) components called for a supply of dry air. A third manifold box contains the hardware to distribute the air into four different volumes. The first two, DME 0/9 and DME 4.5, require dry air to maintain a low level of moisture. The differential pressure between the inside and outside of the (-X) TE is observed with an Omega PX277 Low Pressure Transducer. The next branch feeds air to the LGSWFS CCD window to prevent dust buildup. Finally, air is discharged inside the AOB to maintain a positive pressure. The main flow is adjusted with a Parker regulator and measured with Honeywell AW5104VN Air Flow sensor; each branch may be individually adjusted with a needle valve and cut off with an ASCO solenoid valve.

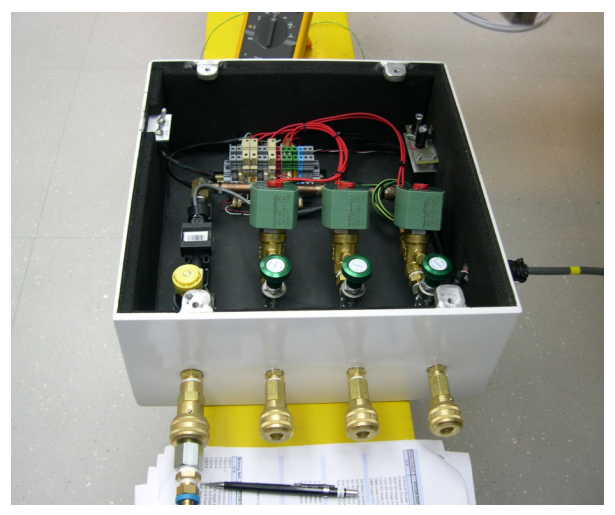

Figure 4. Dry air manifold box is shown dismounted with cover off. Main inlet is at left with three branches at right. 


\section{MONITORING AND CONTROL}

\subsection{TELEMETRY}

At the outcome of several iterations and reviews, the final design for the TE overhaul required the addition of new telemetry capabilities. Several new sensors were added to the system: air and surface temperatures (LM35CZ) at critical locations, dew point temperature sensors (Vaisala DMT142) to mitigate risk of condensation, glycol flow and pressure sensors to detect anomalies in the system, and different binary output line to control power of the DME.

The current implementation accounts for 46 new I/O points added to Canopus. Readout of them was implemented with an IP320A Industrial I/O Pack, 12 Bit Analog Input Board from Acromag installed into a XVME 9660 Industrial I/O Pack Carrier Board. The broadly spaced sensor posed a challenge for noise immunity; this was successfully addressed by having overall shielded cabling Belden 9947 and Belden M 1075A throughout the installation, with a general ground star point at the I/O Interface and analog filters on each of the LM35CZ channels.

\begin{tabular}{|l|c|l|c|}
\hline Device & Number of Units & Function & I/O points \\
\hline Proteus 4012 & 2 & Pressure, Temperature and Flow Sensor & 6 \\
\hline NI-LM35CZ & 12 & Temperature Sensor & 12 \\
\hline Proteus 804 & 7 & Flow Sensor & 7 \\
\hline PT-100 & 1 & Temperature Sensor & 1 \\
\hline Honeywell AWM510 & 1 & Flow Sensor & 1 \\
\hline Vaisala DMT142 & 4 & Dew Temperature Sensor & 4 \\
\hline Omega PX277 & 1 & Differential Pressure Sensor & 1 \\
\hline Ebmpapst 6224NTD & 3 & Adjustable Fan speed & 3 \\
\hline In-house built & 1 & Auto/Manual Coolant Valve status & 1 \\
\hline In-house built & 2 & Auto/Manual Air Valve status & 3 \\
\hline In-house built & 3 & Air Valve control & 2 \\
\hline In-house built & 2 & DME power control & 3 \\
\hline In-house built & 3 & Heartbeat / Reset signals & $\mathbf{4 6}$ \\
\hline
\end{tabular}

Figure5.

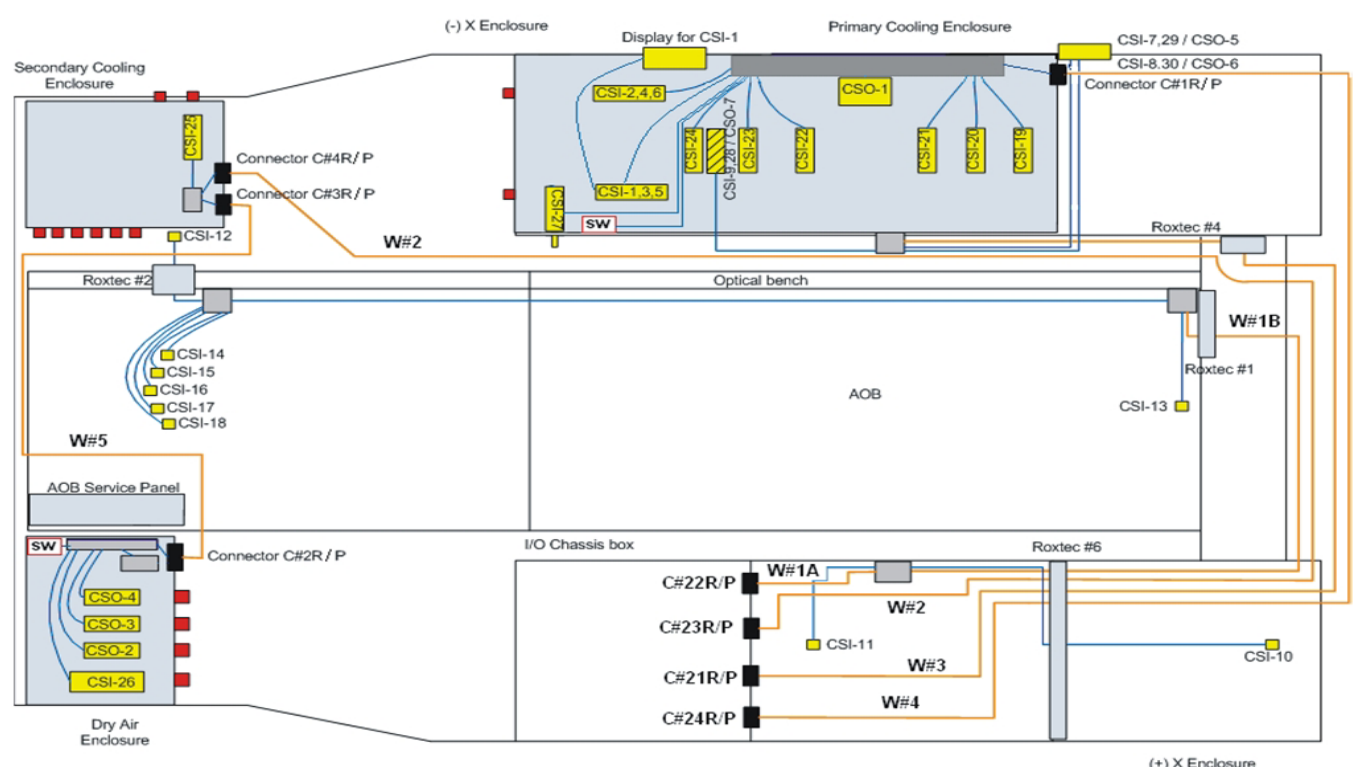

Figure 6. This schematic of CANOPUS underside highlights the various sensors in place between Primary Cooling, Secondary Cooling and Dry Air Enclosures. 


\subsection{ARCHITECTURE}

Canopus opto-mechanical devices are controlled by a VME chassis running vxWorks as real-time operating system and EPIC as general purpose distributed environment. The VME chassis hosts two IPAC carriers supporting up to eight modules in total. Those modules consist of analog input, analog output, binary input and binary output modules from Xycom/Acromag. Free channels on existing modules allowed us to implement the entire IO configuration that was needed.

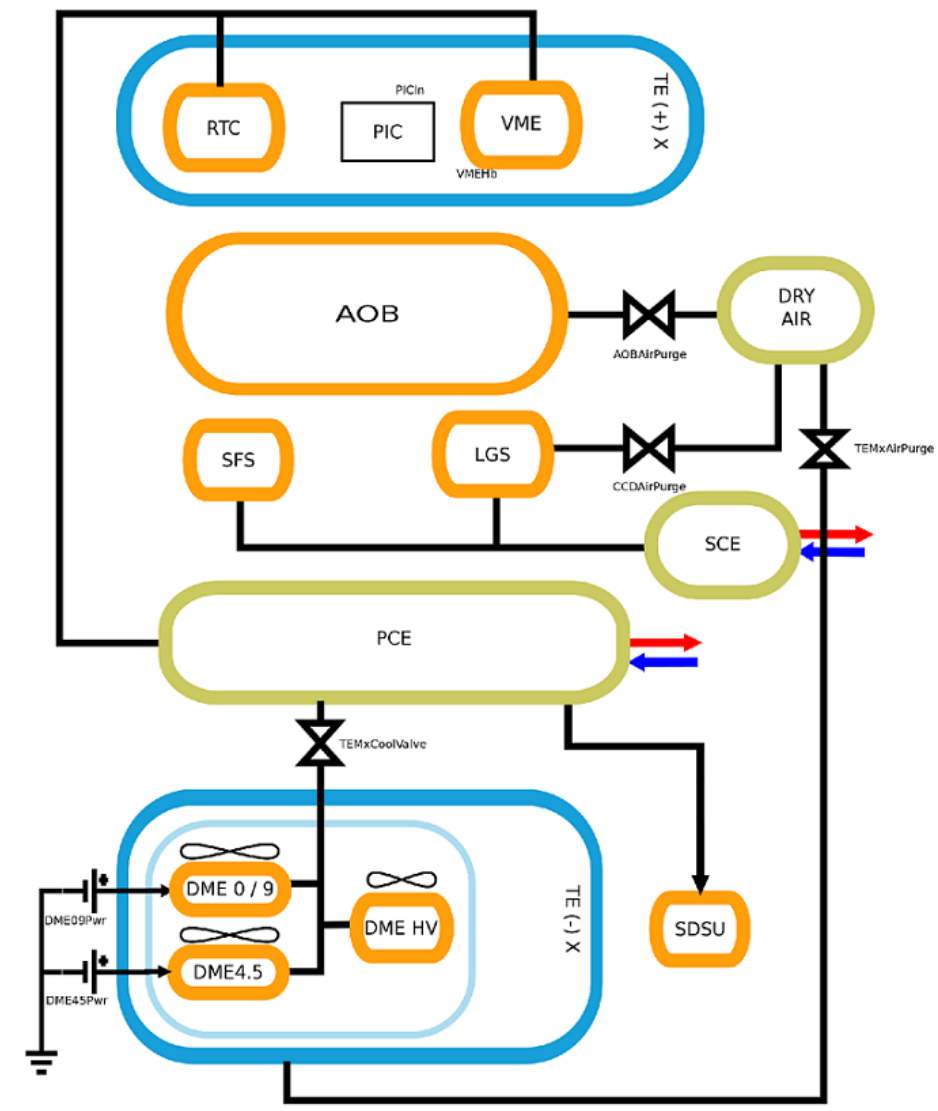

Figure 7. Conceptual diagram of the different units used for the control and monitoring of the system.

\section{Legend}

PCE: Primary Cooling Enclosure (high pressure)

SCE: Secondary Cooling Enclosure (low pressure)

SDSU: San Diego State University Electronics controller

DME and HV: Deformable Mirrors Electronics and High Voltage Power Supplies for Canopus 3 Deformable Mirrors

LGS: Laser Guide Star Wave Front Sensor (mounted on the optical bench)

SFS: Slow Focus Sensor (mounted on the optical bench)

VME: opto-mechs real-time controller

RTC: AO real-time controller 
The input lines are being scanned inside the VME at a configurable rate nominally set to get a new reading every 10 second inside the software. The analog input driver software is configurable to allow averaging a single value from repeated hardware register reading at configurable rate, thus eliminating noise from the reading. In the scope of this project, this was not required but the capability will be present if a sensor becomes noisy and needs some filtering.

\subsection{CONTROL LOGIC}

Each I/O channel is associated in software with nominal range, excursion range and abnormal range. Additionally, those ranges can be configured to report different levels of alarm severity. The control logic takes care of:

- Monitoring all the input signal information and collect new data. The sampling time is configurable per individual channels, typically every 10 seconds; the scan rate was 'cranked up' up to $10 \mathrm{~Hz}$ to help commissioning the sensors electronics and shows no appreciable impact on CPU usage on the VME.

- Comparing all the input values to their individual range and flag their reading value with an associated severity state: no alarm, minor or major alarm.

- Constructing a health status tree by software, combining the individual sensors into sub-unit health, and further combining into a top level health status

- Checking individual piece of program logic check their health and taking appropriate action to regulate the condition or to raise an alarm resulting in powering off the equipment

- The interface to the Gemini Interlock System is used to propagate alarm condition to other sub-systems within MCAO should Canopus enter a critical condition affecting them

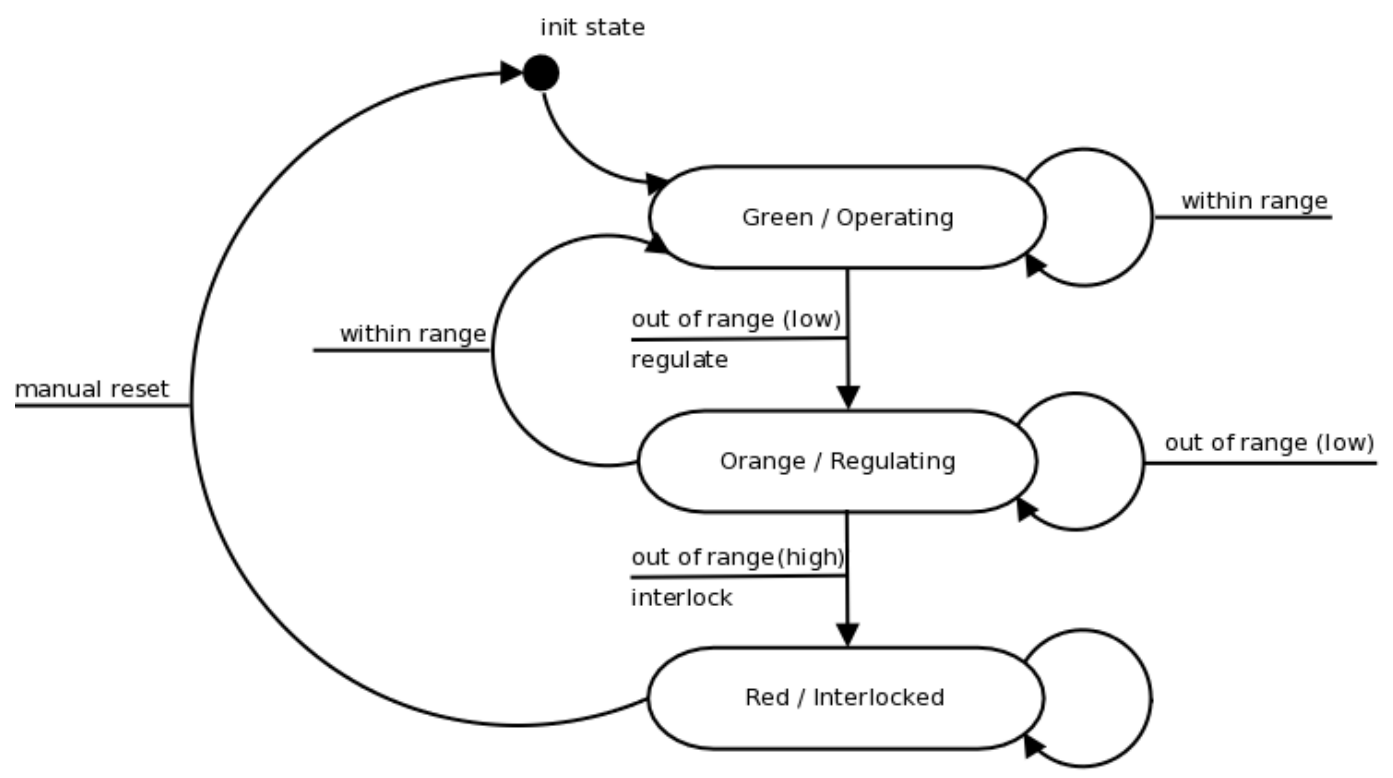

Figure 8. The control logic can be described using a flow diagram. 


\subsection{USER INTERFACE}

The software running on the VME crate uses EPICS $^{[1]}$ as middle ware. EPICS provides a distributed framework for communication over the network. A set of user interfaces have been implemented to provide the user with a convenient overview of the TE data and status. The user interface was developed using Glade ${ }^{[2]}$, a generic GUI builder for GTK $+^{[3]}$ and Python ${ }^{[4]}$. A screen shot of the user interface is presented in Figure 9.

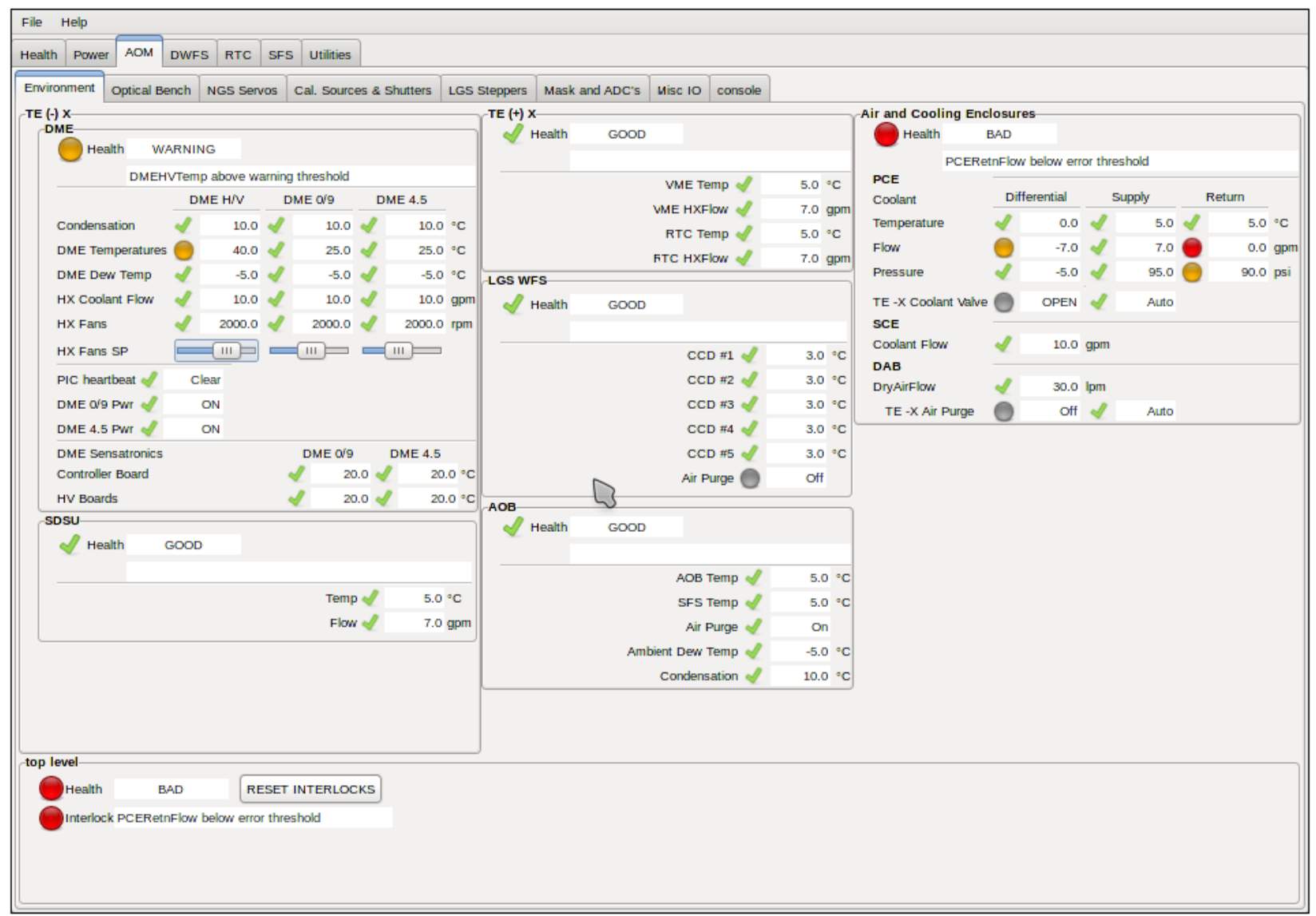

Figure 9. An overview of the monitoring and control capabilities implemented in CANOPUS

In this simulation, the user interface reports the DME HV temperature above warning which is flagged as a minor alarm. The Primary Cooling System Flow sensor reports a low value flagged as an error. Towards the bottom of the screen, the combined state of the system reports an Error state, associated with the highest level from the sub-unit health.

\subsection{ALARM CONDITION HANDLING}

A monitoring system is implemented to take action whenever a subsystem might be damaged by abnormal condition; this normally consists of turning off the electronics equipment and propagating the error to the general Gemini interlock system since Canopus will not be capable of functioning properly. Additionally, the software will prevent the user to reenable a device until it gets back into nominal state and is acknowledged manually with a rest interlock command.

In the case of the DME electronics, protection logic has been put in place to mitigate excursion into warning level. The VME will monitor its input and decide to flush air and adjust the fan's speed in an attempt to recover from a condition without triggering an interlock. 
The DME are powered on with a user issued VME command that is received by a PIC based controller located within the I/O Interface which in turn passes the command through, if and only if, the VME Heart Beat is detected every 2 seconds. In case of an eventual VME failure, causing a heart beat disruption, the PIC controller will start a 5 minutes countdown; if there is no VME heart beat recovery, the PIC controller triggers the shutdown of the DME using the SSR power control box. The design uses CKRD2420 Zero-Crossing Industrial SSRs from Crydom which are very reliable and comply with our fail safe requirement.

\subsection{ARCHIVING}

EPICS is a control system framework widely used at Gemini. A dedicated archiver system, a replica of the one used on site at the telescopes, was deployed in the lab in order to start gathering archive data without affecting the observatory operations. The archiver configuration and data will be migrated to the operation archive when Canopus leaves the lab.

\section{TESTING AND RESULTS}

As of this writing the main functionalities of the system have been tested successfully, first and foremost the ability to cool both Thermal Enclosures at or below the $20 \mathrm{C}$ limit. Through the use of dummy heat sources in a preliminary phase, the system proved able to maintain a lower temperature than anticipated even using slightly higher power than what the design called for.

The major concern in this early testing stage are the vibrations being transmitted to the AOB when operating the large ($\mathrm{X}) \mathrm{TE}$ fans. A coarser correction will be attempted first and the definitive adjustments will happen later once the optic systems are back online.

Once the both TE are fully populated with all the electronic racks, a fine tuning of the different operating parameters will be possible. The control logic will then be tested entirely.

\section{SUMMARY}

CANOPUS is slated to undergo the final stages of preparation during the later part of this year to be ready for full commissioning of GeMS at the telescope in early 2011.

\section{ACKOWLEDGEMENTS}

We thank the valuable effort of all Gemini staff members who contributed in the readiness of CANOPUS System to this date.

The Gemini Observatory is operated by the Association of Universities for Research in Astronomy, Inc, under a cooperative agreement with the NSF on behalf of the Gemini partnership: the National Science Foundation (United States), the Science and Technology Facilities Council (United Kingdom), the National Research Council (Canada), CONICYT (Chile), the Australian Research Council (Australia), Ministério da Ciência e Tecnologia (Brazil) and SECYT (Argentina).

\section{REFERENCES}

[1] EPICS, the Experimental Physics and Industrial Control System http://www.aps.anl.gov/epics/about.php

[2] Glade, a user interface designer for GTK + and Gnome http://glade.gnome.org

[3] GTK+, a highly usable feature rich toolkit for creating graphical user interfaces http://www.gtk.org

[4] Python, a dynamic object-oriented programming language http://www.python.org 